Ann. Biol. anim. Bioch. Biophys., 1978, 18 (2A), 237-247.

\title{
Mesure du transit gastrointestinal chez le porc à l'aide des radiolanthanides. Comparaison avec le mouton
}

\author{
par Marie-France THIELEMANS *, E. FRANÇOIS **, C. BODART * , A. THEWIS *** \\ Avec la collaboration technique de F. DUPONT **, L. LALLEMAND **, C. MALBURNY* \\ * Station de Zootechnie \\ ** Station de Chimie et de Physique Agricoles \\ Centre de Recherches Agronomiques de l'Ełat, Gembloux, Belgique \\ *** Chaire de Physiologie animale ef de Zoofechnie, \\ Faculté des Sciences Agronomiques de l'Etał, Gembloux, Belgique
}

Summary. Gastrointestinal transit in the pig : measurement using radioactive lanthanides and comparison with sheep.

A technique to characterize the transit time of digesta through the gastrointestinal tract of the sheep has been described by François and Compere (1971). In the present paper, the authors present an adaptation of the method to the pig. On the first day of the experiment, each animal was given a diet uniformly labelled with ${ }^{144} \mathrm{Ce}$ as an unabsorbable marker. The feces were collected at 1 or 2-hour intervals at progressively extended periods for the remainder of the collection period. The fecal concentration of the marker rose rapidly to a maximum, then decreased following a simple exponential law. Data were also expressed as a cumulative excretion curve of ${ }^{144} \mathrm{Ce}$. The mean retention time in the pig digestive tract could be calculated by concentration curves using either the $m_{t}$ parameter of Debouche (1974) or the $\bar{f}$ parameter proposed by Levenspiel (1974) to describe the flow of a fluid in an open vessel. The values obtained were identical to those found by Castle and Castle (1957) for the $R$ parameter. These data are compared to those given by the same technique with the sheep.

\section{Introduction.}

Si la rentabilité de l'élevage et de l'engraissement dépend de la composition et du prix de la ration, elle dépend également de l'efficacité d'utilisation des aliments ; cette dernière est notamm€nt liée au temps de séjour des particules alimentaires dans le tube digestif (Aumaitre et al., 1969 ; Rérat, 1971). Dès lors, il n'est pas étonnant que de nombreux chercheurs se soient efforcés d'estimer ce paramètre.

A cette fin, les traceurs non résorbables sont le plus souvent utilisés. Chez le Porc, parmi les marqueurs employés, on peut citer le polyéthylène glycol (Ishikawa, 1966 ; Ishikawa et Furuichi, 1968 ; Manners ef Kidder, 1967 ; Furuya ef Takahashi, 1975), marqueur de la phase liquide, l'oxyde de chrome (Cunningham, 1967 ; Eeckhout, 
1972 ; Lawrence, 1970a ef $b$; Furuya ef Takahashi, 1975), l'oxyde de fer (Entringer et al., 1975 ; Maner et al., 1966), certains colorants (Castle et Castle, 1956, 1957 ; Keys et De Barthe, 1974b), marqueurs de la phase solide.

Les travaux réalisés sur les ruminants (François, Compère ef Rondia, 1968 ; François et Compère, 1971) ont permis de démontrer que parmi les traceurs proposés, les lanthanides et, en particulier, certains de leurs radioisotopes réunissent le plus de qualités, tant au point de vue du marquage que de la facilité de mise en œuvre ef de détermination. Le radiocérium a été utilisé dans des travaux antérieurs pour étudier le transit des résidus de la digestion chez le Mouton et le Rat (François, Compère et Rondia, 1968). Une méthode basée sur l'étude des courbes caractérisant l'excrétion par l'évolution de la concentration fécale en ${ }^{144} \mathrm{Ce}$ a été mise au point (François et Compère, 1971). L'application de cette technique au Porc, la comparaison des renseignements obtenus à certains paramètres analogues mentionnés dans la littérature (Castle et Castle, 1956) et aux données antérieures précisées sur moutons font l'objet du présent travail.

\section{Matériel expérimental et techniques utilisées.}

Porcs.

Animaux ef alimentation. - Les animaux utilisés sont des porcs castrés Landrace Belge. Au poids de $30 \mathrm{~kg}$, ils sont accoutumés aux cages à métabolisme permettant la récolte séparée des fèces et des urines. L'aliment est distribué sous forme de bouillie deux fois par jour à $9 \mathrm{~h}$ et $16 \mathrm{~h}$. En un quart d'heure, la totalité de la ration est consommée. L'eau de boisson est présentée à $13 \mathrm{~h}$ et directement après les repas. La consommation d'eau n'est pas limitée.

Administration du radiocérium. - La masse de farine nécessaire à la préparation des rations individuelles marquées est étalée dans une hotte de laboratoire munie d'une ventilation adéquate. Elle est alors traitée par nébulisation en plusieurs passages de la solution radioactive. Cette solution a été préalablement légèrement acidifiée par de l'acide chlorhydrique afin d'éviter l'adsorption du radiocérium sur les surfaces venant en contact avec elle. Chaque passage est suivi d'un séchage aux rayons infra rouges. Afin d'obtenir un marquage uniforme, la farine est alors mélangée mécaniquement (mélangeur en $V$ ). Les rations individuelles sont préparées à partir de la masse ainsi marquée. Chaque animal reçoit la ration radioactive le premier jour de l'essai lors du repas de $9 \mathrm{~h}$.

Transit gastrointestinal. - Les récoltes quantitatives de matière fécale sont effectuées toutes les heures ou toutes les deux heures à partir de l'administration du repas marqué pour s'espacer progressivement jusqu'au 6e jour. Les échantillons de fèces sont séchés à $100^{\circ} \mathrm{C}$ jusqu'à poids constant. Ils sont pesés, finement moulus et soumis à l'analyse radiochimique.

Défermination radiochimique. - La concentration en ${ }^{{ }^{144}} \mathrm{Ce}-{ }^{144} \mathrm{Pr}-$ couple de radionuclides en filiation - esł déterminée à partir de leur émission bêta comme décrit par Thewis, François et Thill (1975) à l'aide d'un détecteur à gaz, à fenêtre mince et à faible mouvement propre ( $1 \mathrm{CPM})$. Un poids fixe de matière fécale moulue 
- 4,5 g dans le cas présent - est comprimé en disques à la presse hydraulique dans une matrice. Ces derniers résistent parfaitement aux manipulations.

Expériences individuelles. - Huit porcs d'un poids moyen de $35 \mathrm{~kg}$ sont utilisés (Exp. I) ; durant toute l'expérience, les animaux reçoivent deux fois par jour $700 \mathrm{~g}$ de farine additionnée à 1 I d'eau. La teneur de l'aliment en protéine brute est de 18 p. 100 , celle en cellulose (Weende) de 9 p. 100 . Au poids de $65 \mathrm{~kg}$, les mêmes porcs font l'objet d'une deuxième détermination de la vitesse de transit (Exp. II). Une farine de composition différente (15 p. 100 de protéine brute, 6 p. 100 de cellulose) est employée. Chaque repas est constitué de $1200 \mathrm{~g}$ de farine mélangée à 1 I 500 d'eau. Il est également distribué deux fois par jour. L'emploi de régimes différents donne une portée plus générale aux observations. Le tableau 1 reprend pour les deux essais les poids et indices d'ingestion $\left({ }^{1}\right)$ des porcs.

TABLEAU 1

Poids (en $\mathrm{kg}$ ) et indices d'ingestion (I) * des porcs

\begin{tabular}{cccccc}
\hline & \multicolumn{2}{c}{ Expérience I } & & \multicolumn{2}{c}{ Expérience II } \\
\cline { 2 - 3 } \cline { 5 - 6 } Porc no & $\begin{array}{c}\text { Poids } \\
\text { vif }\end{array}$ & $\begin{array}{c}\text { Indice } \\
\text { d'ingestion }\end{array}$ & & $\begin{array}{c}\text { Poids } \\
\text { vif }\end{array}$ & $\begin{array}{c}\text { Indice } \\
\text { d'ingestion }\end{array}$ \\
\hline & 34,25 & 84,07 & & 65,50 & 90,21 \\
1 & 39,65 & 75,31 & & 70,60 & 85,28 \\
2 & 40,60 & 73,99 & & 62,15 & 93,83 \\
3 & 31,90 & 89,65 & & 64,50 & 91,26 \\
4 & 36,55 & 80,04 & & 67,50 & 88,20 \\
5 & 31,05 & 90,46 & & 55,75 & 101,80 \\
6 & 33,40 & 85,64 & & 67,60 & 88,10 \\
7 & 31,09 & 90,37 & & 62,15 & 93,83 \\
8 & & & & & \\
\hline
\end{tabular}

$* \mathrm{I}=\frac{\text { consommation de matière sèche }(\mathrm{kg})}{\text { poids } 0,75(\mathrm{~kg})} \times 1000$.

Béliers.

Nous reprenons d'une publication antérieure (Thewis ef al., 1976) les données relatives aux animaux $n^{\text {os }} \mathbf{4 7 9}$ ef 498 . Rappelons que ces béliers recevaient du foin de prairie haché. Le tableau 2 reprend la consommation de matière sèche, le poids et l'indice d'ingestion des deux sujets. Les animaux ont reçu une gélule de ${ }^{144} \mathrm{Ce}$ et ont fait l'objet d'une mesure de vitesse de transit suivant François ef Compère (1971).

Traitement des données. - L'excrétion du radiocérium peut être représentée par la courbe d'excrétion cumulée du traceur ou par la courbe de concentration. Elles permettent de définir cerfains paramètres.

(1) $\mathrm{I}=\frac{\text { Consommation de matière sèche }(\mathrm{kg})}{\text { Poids } 0,75(\mathrm{~kg})} \times 1000$. 
TABLEAU 2

Consommation journalière $(\mathrm{en} \mathrm{g})$, poids $(\mathrm{en} \mathrm{kg})$ et indice d'ingestion $(\mathrm{l})$ * des moutons

\begin{tabular}{cccc}
\hline Mouton $\mathrm{n}^{\circ}$ & $\begin{array}{c}\text { Consommation } \\
\text { journalière } \\
\text { matière sèche }\end{array}$ & $\begin{array}{c}\text { Poids } \\
\text { vif }\end{array}$ & $\begin{array}{c}\text { Indice } \\
\text { d'ingestion }\end{array}$ \\
\hline 479 & 885 & 53,0 & 45,05 \\
498 & 1123 & 58,5 & 53,09 \\
\hline
\end{tabular}

$* I=\frac{\text { consommation de matière sèche }(\mathrm{kg})}{\text { poids }^{0,75}(\mathrm{~kg})} \times 1000$.

1) Courbe d'excrétion cumulée : La quantité de marqueur excrété depuis le moment de l'administration (origine des temps) est décrite par une courbe du type sigmoïde. Cette courbe permet de déterminer graphiquement le temps de séjour moyen à l'aide du paramètre $R$ défini par Castle et Castle (1956). Elle peut également servir à définir les temps nécessaires à l'excrétion de 5 et de 95 p. 100 du traceur ingéré ainsi que leur différence $\left(t_{95}\right.$ p. $100-t_{5}$ p. 100 $)$.

2) Courbe de concentration : A partir des courbes décrivant l'évolution de la concentration fécale en traceur (François et Compère, 1971) et grâce au modèle mathématique de Debouche (1974), on calcule un temps moyen de séjour $\left(m_{t}\right)$ du marqueur dans le tractus digestif et un temps $t_{0}$ (temps écoulé entre l'ingestion du marqueur et l'apparition des premières particules marquées).

Dans ce modèle mathématique, la concentration en ${ }^{144} \mathrm{Ce}$ est assimilée à la fréquence observée du nombre de particules qui ont traversé le tube digestif en un temps $\dagger_{\mathbf{i}}$. Une fonction gamma à 4 paramètres peut s'ajuster (moindres carrés) sur ces fréquences observées et permet alors de calculer facilement l'espérance mathématique de la variable c'est-à-dire le temps moyen de passage. L'équation de la fonction gamma est la suivante :

$$
y=a\left(t-t_{0}\right)^{b} \cdot e-c\left(t-t_{0}\right)
$$

où y est la concentration en ${ }^{144} \mathrm{Ce} ; \dagger$ est le temps; $\dagger_{0}$ le temps écoulé avant l'excrétion des premières particules marquées ; $a, b, c$ sont les autres paramètres du modèle.

Le temps moyen de passage, $m_{t}$, est égal à $\frac{b+1}{c}+t_{0}$.

Par ailleurs, sans faire appel à un modèle mathématique et à condition de disposer d'un nombre suffisant d'observations réparties sur toute la période d'excrétion, il esł possible d'obtenir une approximation du temps moyen de séjour (ఫ) plus proche de la réalité dans certains cas, par la méthode des moments. La relation utilisée est du type :

$$
\overline{\mathbf{i}}=\frac{\Sigma \mathrm{t}_{\mathrm{i}} C_{\mathbf{i}} \Delta \mathbf{t}_{\mathbf{i}}}{\Sigma C_{\mathbf{i}} \Delta \mathrm{t}_{\mathbf{i}}}
$$

où $C_{i}$ représente la concentration en marqueur au temps $t_{1}$ dans la matière fécale récoltée pendant $\Delta t_{i}$ heures. 
Levenspiel (1974) étudiant le flux des fluides dans les réacteurs utilisés en génie chimique et analysant de la même manière l'évolution de la concentration d'un marqueur après injection d'une dose unique en avant du système, propose un modèle particulier d'expression des résultats. Le temps est exprimé en fonction du temps moyen de séjour, $\theta=t / \bar{t}$; la concentration est calculée suivant la formule

$$
C \theta_{i}=\frac{C_{i}}{\Sigma C_{i} \Delta \theta_{i}}
$$

Alors, l'aire comprise sous la courbe est unitaire.

Cette standardisation des courbes facilite leur comparaison : les courbes obtenues s'écartent, en se déformant, d'autant plus de la courbe de Gauss symétrique (axée sur la droite $\theta=1$ ) que la dispersion du fluide au cours de son transit est importante. De plus, on peut définir pour les digesta un coefficient $D / u L$ chiffrant la dispersion axiale d'écoulement où $D$ est le coefficient de dispersion axiale ou longitudinale ; $L$ la longueur de déplacement ; u la vitesse constante du fluide.

\section{Résultats.}

Le tableau 3 donne pour les deux expériences réalisées sur porcs les paramètres

TABLEAU 3

Paramètres dérivés des courbes d'activité cumulée ef de concentration en traceur dans les matières fécales ( ${ }^{1}$ ) - Porcs

\begin{tabular}{|c|c|c|c|c|c|c|c|c|}
\hline & $\begin{array}{c}\text { Porc } \\
n^{\circ}\end{array}$ & $t_{5 p \cdot 100}$ & $t_{95} p \cdot 100$ & $t_{95} p \cdot 100-t_{5} p \cdot 100$ & $\mathbf{R}$ & $\bar{t}$ & $\mathrm{~m}_{\mathrm{t}}$ & $\dagger_{0}$ \\
\hline Exp. I & $\begin{array}{l}1 \\
2 \\
3 \\
4 \\
5 \\
6 \\
7 \\
8\end{array}$ & $\begin{array}{r}17,40 \\
17,00 \\
12,00 \\
8,00 \\
13,30 \\
10,00 \\
11,40 \\
10,40\end{array}$ & $\begin{array}{l}46,40 \\
50,00 \\
46,42 \\
36,00 \\
51,20 \\
49,10 \\
40,40 \\
43,20\end{array}$ & $\begin{array}{l}29,00 \\
33,00 \\
34,42 \\
28,00 \\
37,50 \\
39,10 \\
29,00 \\
33,40\end{array}$ & $\begin{array}{l}28,27 \\
32,47 \\
25,55 \\
21,07 \\
29,48 \\
25,29 \\
23,36 \\
25,39\end{array}$ & $\begin{array}{l}29,57 \\
32,26 \\
26,20 \\
21,49 \\
30,52 \\
26,34 \\
24,17 \\
25,32\end{array}$ & $\begin{array}{l}29,20 \\
34,16 \\
26,28 \\
21,55 \\
32,02 \\
27,01 \\
24,11 \\
25,04\end{array}$ & $\begin{array}{l}6,44 \\
7,04 \\
5,19 \\
3,19 \\
6,02 \\
4,08 \\
5,46 \\
2,42\end{array}$ \\
\hline $\begin{array}{l}\bar{x} \\
\text { D. S. } \\
\text { C. V. (p. 100) }\end{array}$ & & $\begin{array}{r}12,34 \\
3,21 \\
26,69\end{array}$ & $\begin{array}{r}45,29 \\
5,11 \\
11,42\end{array}$ & $\begin{array}{r}32,31 \\
4,10 \\
12,65\end{array}$ & $\begin{array}{r}26,36 \\
3,40 \\
13,74\end{array}$ & $\begin{array}{r}27,13 \\
3,35 \\
13,16\end{array}$ & $\begin{array}{r}27,32 \\
4,08 \\
14,99\end{array}$ & $\begin{array}{r}5,08 \\
1,35 \\
31,00\end{array}$ \\
\hline Exp. II & $\begin{array}{l}1 \\
2 \\
3 \\
4 \\
5 \\
6 \\
7 \\
8\end{array}$ & $\begin{array}{r}11,50 \\
17,20 \\
13,10 \\
9,40 \\
15,20 \\
12,50 \\
10,10 \\
10,30\end{array}$ & $\begin{array}{l}38,30 \\
45,00 \\
33,00 \\
30,50 \\
39,00 \\
32,30 \\
34,40 \\
35,30\end{array}$ & $\begin{array}{l}26,40 \\
28,10 \\
19,50 \\
21,10 \\
23,40 \\
19,30 \\
24,30 \\
25,00\end{array}$ & $\begin{array}{l}24,10 \\
30,16 \\
23,09 \\
19,36 \\
27,04 \\
23,44 \\
22,00 \\
23,06\end{array}$ & $\begin{array}{l}24,32 \\
30,49 \\
23,52 \\
20,37 \\
26,48 \\
23,32 \\
21,21 \\
23,40\end{array}$ & $\begin{array}{l}25,07 \\
30,55 \\
24,08 \\
20,13 \\
26,27 \\
24,20 \\
21,36 \\
23,56\end{array}$ & $\begin{array}{r}4,08 \\
2,42 \\
2,52 \\
1,22 \\
3,05 \\
12,31 \\
5,23 \\
2,22\end{array}$ \\
\hline $\begin{array}{l}\bar{x} \\
\text { D. S. } \\
\text { C. V. (p. 100) }\end{array}$ & & $\begin{array}{r}12,36 \\
2,40 \\
21,14\end{array}$ & $\begin{array}{r}36,06 \\
4,35 \\
12,71\end{array}$ & $\begin{array}{r}23,30 \\
3,04 \\
13,03\end{array}$ & $\begin{array}{r}24,08 \\
3,14 \\
13,43\end{array}$ & $\begin{array}{r}24,24 \\
3,13 \\
13,15\end{array}$ & $\begin{array}{r}24,35 \\
3,13 \\
13,09\end{array}$ & $\begin{array}{r}4,11 \\
3,37 \\
86,70\end{array}$ \\
\hline
\end{tabular}

(1) Les temps sont exprimés en heures ef minutes. 
déterminés à partir des courbes d'excrétion cumulée et de celles décrivant l'évolution de la concentration fécale en ${ }^{144} \mathrm{Ce}$. Les varictions individuelles des différents paramètres sont faibles. Néanmoins, des coefficients de variation plus élevés sont observés pour $t_{0}$ et $t_{5}$ p. 100, ces temps étant liés aux premières défécations. L'analyse de la variance réalisée sur les deux séries de valeurs de $R, m_{t}$ et $\bar{f}$ ne mel pas en évidence une différence significative. Une légère diminution est observée au cours de la seconde expérience.

A titre d'exemple, la courbe obtenue pour le porc no 7 (Exp. I) est présentée à la figure 1.
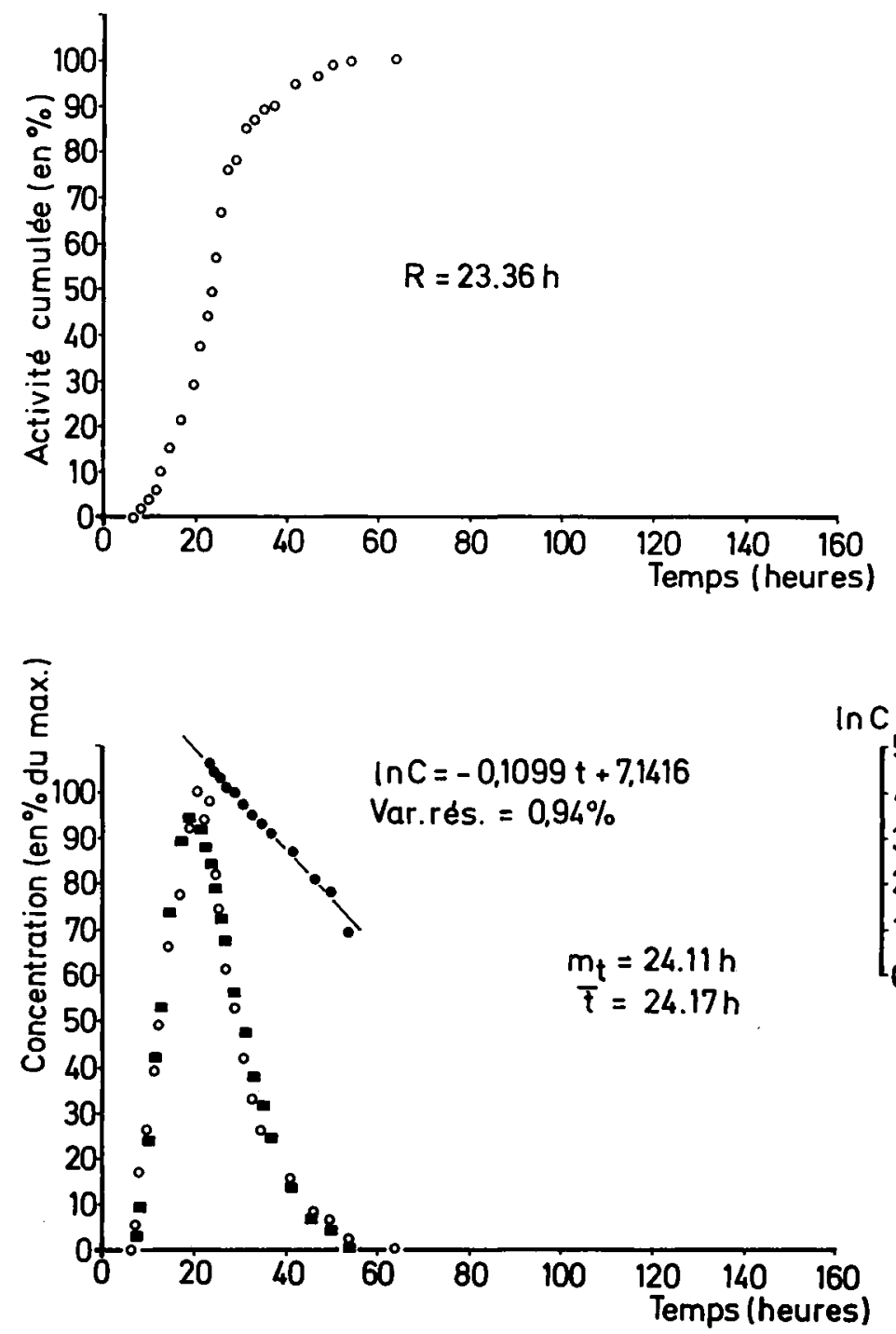

FIG. 1. - Excrétion d'un repos marqué uniformément au ${ }^{144} \mathrm{Ce}$ chez le Porc (Sujet 7, Exp. I). - Valeurs observées ; - Valeurs théoriques (Debouche, 1974). 
TABLEAU 4

Paramètres dérivés des courbes d'activité cumulée et de concentration en fraceur dans les matières fécales $\left(^{1}\right)$ - Moutons

\begin{tabular}{|c|c|c|c|c|c|c|c|}
\hline $\begin{array}{c}\text { Mouton } \\
n^{0}\end{array}$ & $t_{5 p \cdot 100}$ & $t_{95} \cdot 100$ & $t_{y 5 \cdot 100}-t_{5 p \cdot 100}$ & $\mathbf{R}$ & $\bar{i}$ & $m_{t}$ & $t_{0}$ \\
\hline $\begin{array}{l}479 \ldots \ldots \\
498 \ldots \ldots\end{array}$ & $\begin{array}{l}18,00 \\
15,50\end{array}$ & $\begin{array}{l}111,00 \\
108,00\end{array}$ & $\begin{array}{l}93,00 \\
92,50\end{array}$ & $\begin{array}{l}42,35 \\
44,47\end{array}$ & $\begin{array}{l}48,52 \\
47,53\end{array}$ & $\begin{array}{l}45,01 \\
42,32\end{array}$ & $\begin{array}{l}11,24 \\
10,23\end{array}$ \\
\hline
\end{tabular}

(1) Les temps sont exprimés en heures et minutes.
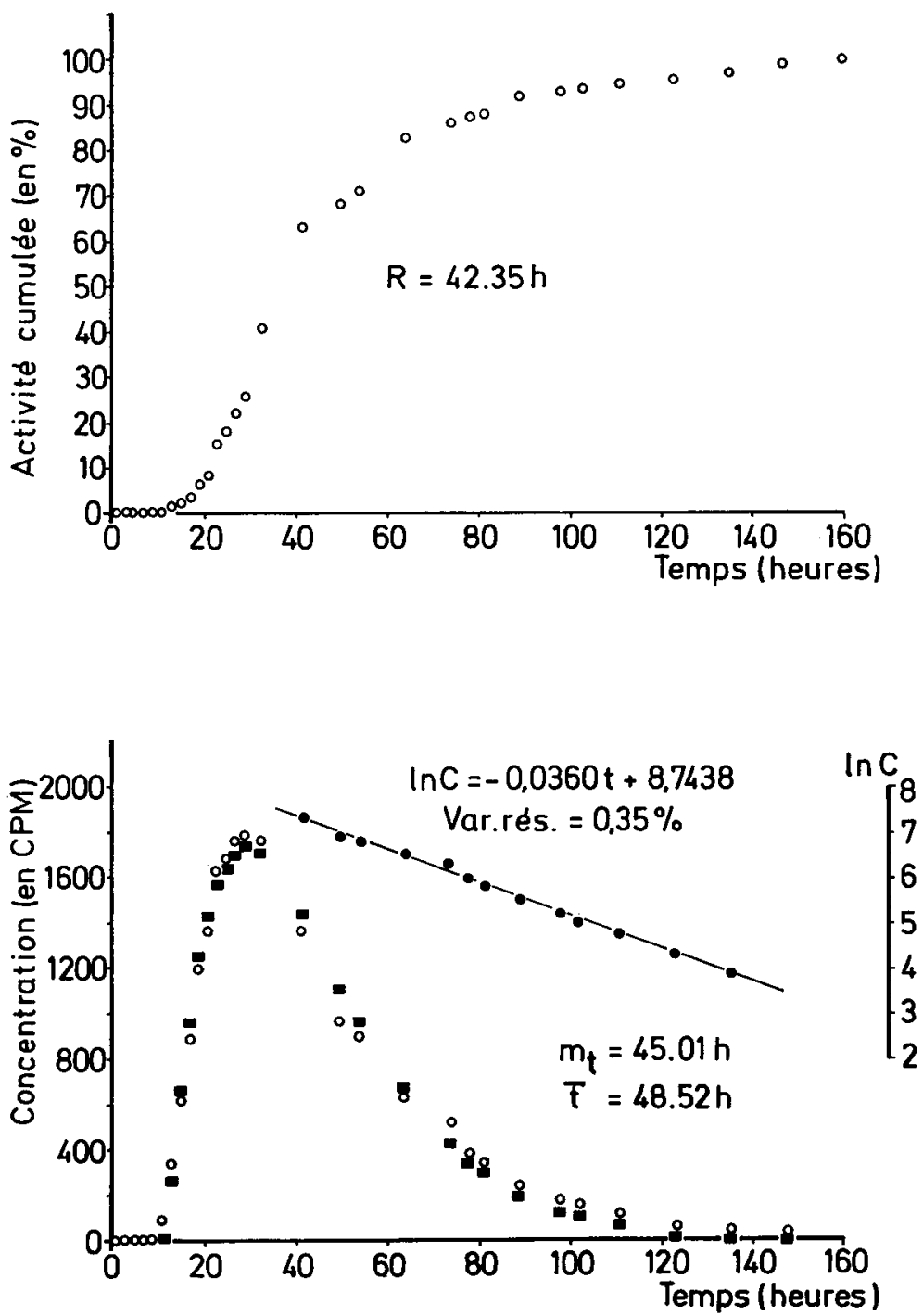

FIG. 2. - Excrétion du ${ }^{144} \mathrm{Ce}$ administré par gélule chez le Mouton (Sujet 479).

- Valeurs observées ; - Valeurs théoriques (Debouche, 1974). 
Pour permettre une discussion comparée entre le Porc et le Mouton, les valeurs $R, \bar{t}$ et $m_{t}$ des deux moutons envisagés sont notées au tableau 4. La courbe se rapportant au mouton no $\mathbf{4 7 9}$ fait l'objet de la figure 2.

Nous donnons à la figure 3 les courbes de 3 porcs et de 2 moutons en coordonnées réduites selon la méthode de Levenspiel (1974). Une dissymétrie plus prononcée apparaît chez le Mouton.

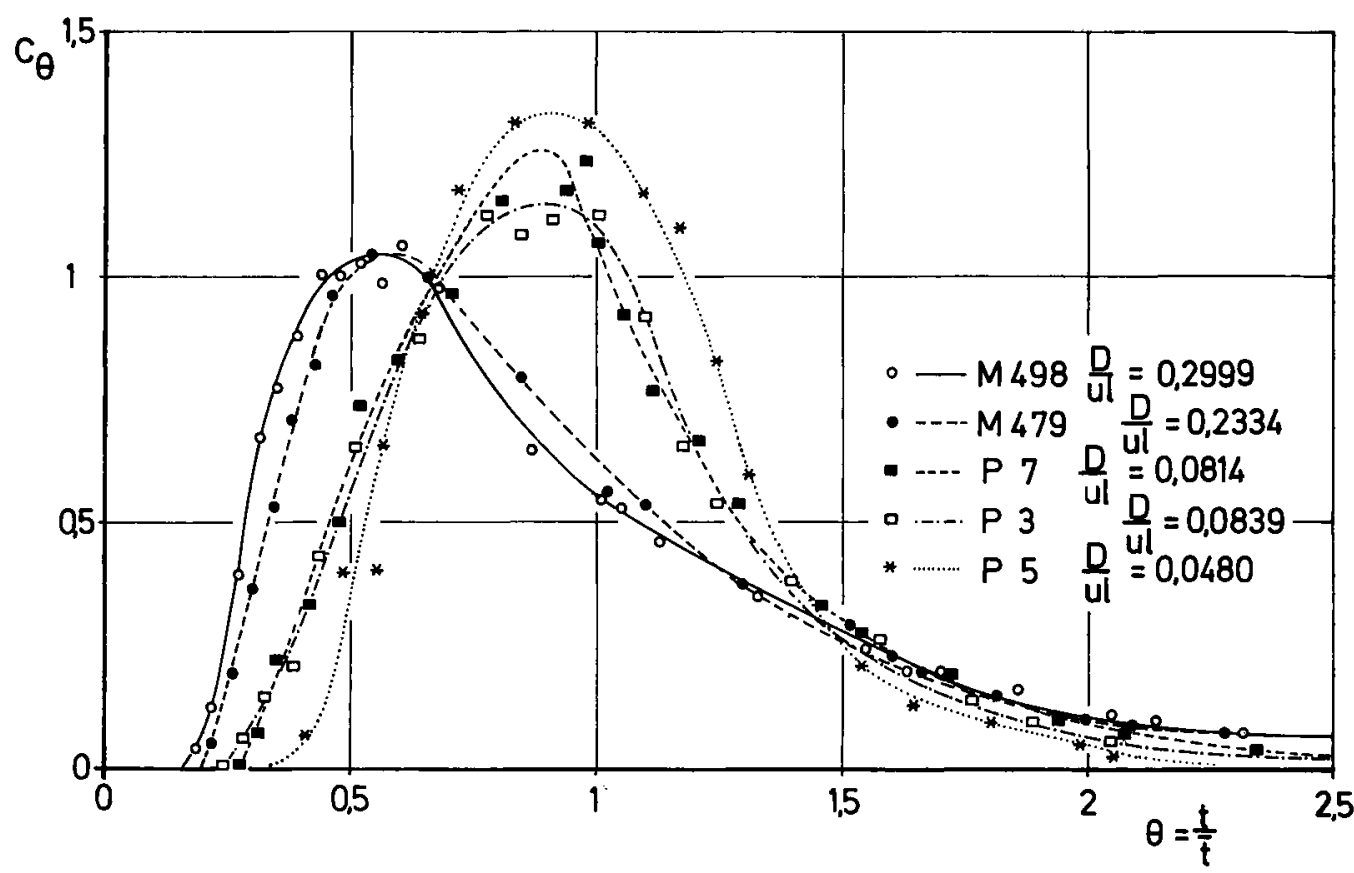

FIG. 3. - Courbes de concentration en coordonnées réduites suivant la méthode de Levenspiel (1974). $\mathrm{M}=$ Mouton; $\mathrm{P}=$ Porc.

\section{Discussion.}

On voit qu'il est possible de caractériser le transit digestif du Porc, tout comme celui du Mouton (François, Compère et Rondia, 1968 ; François et Compère, 1971), par l'étude de l'excrétion d'une dose unique d'indicateur non résorbable. Cependant, la courbe caractérisant l'excrétion du marqueur ne peut se comparer à celle obtenue chez le Mouton que si le Porc reçoit un repas intégralement et uniformément marqué. Dans ces conditions, la phase décroissante de la courbe de concentration fécale peut être assimilée également à une exponentielle. II est vraisemblable que les irrégularités observées dans l'excrétion du marqueur chez le Rat (François, Compère et Rondia, 1968) et chez le Porc (Thewis, données non publiées) lorsque le repas n'est pas intégralement marqué proviennent de ce que le mélange du contenu est moins intense dans l'estomac que dans le rumen. Cet auteur administrant le traceur adsorbé sur une petite quantité de farine contenue dans une gélule, obtient des courbes d'excrétion d'une allure générale semblable à celle des courbes présentées dans ce travail. 
Mais la branche décroissante des courbes d'excrétion du marqueur présentait un pic secondaire, correspondant à l'excrétion d'une fraction de contenu à concentration plus élevée. Chez le Mouton, par contre, les contractions du réticulo-rumen assurant un brassage efficace de son contenu permettent d'administrer la dose de radiocérium indifféremment sous la forme d'une fraction de fourrage marqué dont on veut étudier le transit ou d'une gélule contenant le marqueur fixé sur un aliment concentré (Ellis et Huston, 1968). Dans ces deux cas, ce marqueur libéré des particules de support aisément digérées, se fixe sur les constituants de la ration de base (Poncet, 1976). Par suite de ce marquage secondaire, et dans des conditions de brassage efficace, les courbes d'excrétion observées ne diffèrent pas significativement. Dans ces conditions, d'après Ellis et Huston (1968), il n'est pas possible d'étudier sélectivement le transit de particules de dimensions données ; il serait, d'après ces auteurs, déterminé principalement par le flux d'une masse plus importante de résidus de la ration de base, plutôt que par les propriétés intrinsèques de ces particules.

Chez le Porc, l'allure peu dissymétrique des courbes d'excrétion du radiocérium consécutives à l'ingestion d'un repas uniformément et intégralement marqué laisse supposer que ce repas se mélange relativement peu aux résidus des repas antérieurs et à ceux des suivants. Le marquage secondaire certainement présent ne servirait, dans ces conditions, qu'à parfaire la répartition du cérium au sein du repas marqué.

Les travaux réalisés sur porcs par Auffray, Martinet et Réraf (1967) ef par Laplace ef Tomassone (1970) montrent une évacuation rapide du contenu stomacal. Celle-ci se traduit par des valeurs de paramètres plus faibles que chez le polygastrique.

En ce qui concerne l'allure des courbes de concentration, la représentation proposée par Levenspiel (1974) permet d'utiles comparaisons entre le Mouton et le Porc. Remarquons cependant que si on ne peut assimiler le tube digestif à un ensemble inerte de tuyaux et de réservoirs, les courbes de concentration obtenues pour ce système dans des conditions analogues, après injection d'une dose unique de traceur, sont tellement comparables à celles qui caractérisent l'excrétion d'une dose unique de radiocérium chez le Porc que cette représentation semble instructive. Les valeurs de $D / u L$, sans dimensions, reportées à la figure 3 concrétisent le fait que les courbes d'excrétion fécale du radiocérium chez le Porc sont visiblement moins étalées que chez le Mouton ; elles s'écartent moins de la courbe de Gauss symétrique, axée sur $\theta=1$, caractéristique des systèmes à faible degré de dispersion. La dispersion axiale beaucoup moindre observée dans le tube digestif, du Porc, correspondant à des valeurs de $\mathrm{D} / \mathrm{uL}$ plus faibles, traduit vraisemblablement la plus grande complexité du tube digestif du Mouton.

Chez le Porc, $m_{t}$ et $\bar{t}$ sont identiques ; le modèle de Debouche (1974) se superpose en effet parfaitement à la courbe observée.

Chez le Mouton, l'écart observé essentiellement dans les « queves » des courbes détermine une certaine différence entre les deux paramètres $\left(m_{t}\right.$ et $\left.\bar{t}\right)$. Si, en règle générale, $R$ nous donne une bonne approximation du temps moyen de rétention (tabl. 3, voir Thewis et al., 1976), sa détermination, ainsi que celle de $t_{5}$ p. $\mathbf{1 0 0}_{\mathbf{1 0}}$ et de $t_{95} \mathrm{p} \cdot{ }_{100}$ exige une récolte totale des fèces pendant toute la période expérimentale. Le temps $t_{5}$ p ${ }_{100}$ présente de plus l'inconvénient de dépendre fortement du moment des premières défécations. Le temps moyen obtenu par la méthode de Levenspiel (1974) est de calcul aisé et nous semble la meilleure mesure du temps moyen de séjour 
pour autant que l'on dispose d'un nombre suffisant de récoltes sur toute l'étendue de la courbe de concentration en marqueur. Faichney (1975) propose chez le Mouton une méthode de calcul semblable mais la variable étudiée est le pourcentage de traceur excrété. Cette méthode donne des courbes irrégulières du fait des fluctuations dans l'excrétion de la matière sèche.

Nous voyons donc que, dans les conditions d'administration choisies, l'étude de l'excrétion du radiocérium réparti uniformément permet également de définir très simplement chez le Porc un temps de séjour moyen de ce marqueur dans le tractus digestif.

Reçu en août 1977.

Accepté en octobre 1977.

\section{Références}

AUFFRAY P., MARTINET J., RERAT A., 1967. Quelques aspects du transit gastrointestinal chez le porc. Ann. Biol. anim. Bioch. Biophys., 7, 261-279.

AUMAITRE A., FÉVRIER C., RÉRAT A., RIGAUD J., THIVEND P., 1969. Application de l'analyse en continu à l'éfude des variations de la glycémie du sang porte au cours de la digestion chez le porc. C. R. Acad. Sci. Paris, Sér. D, 268, 717-720.

CASTLE E. J., CASTLE M. E., 1956. The rate of passage of food through the alimentary tract of pigs. J. agric. Sci., 47, 196-203.

CASTLE E. J., CASTLE M. E., 1957. Further studies of the rate of passage of food through the alimentary tract of pigs. J. agric. Sci., 49, 106-112.

CUNNINGHAM H. M., 1967. Digestibility, rate of passage and rate of gain in the gastrectomized pig. J. Anim. Sci., 26, 500-503.

DEBOUCHE C. L. L., 1974. Etude d'un modèle mathématique empirique pour la mesure du transit gastrointestinal. Bull. Rech. agron., Gembloux, 9, 335-342.

EECKHOUT W., 1972. Vitesse de passage de granulés et de farine à travers le tube gastrointestinal des porcs Landrace belge et Piétrain. Revue de l'Agriculture, 3, 421-433.

ELLIS W. C., HUSTON J. E., 1968. ${ }^{144} \mathrm{Ce}^{144} \mathrm{Pr}$ as a particulate digesta flow marker in ruminants. J. Nutr., 95, 67-68.

ENTRINGer R. P., PLUMLeE M. P., CONRAD J. H., CLINE T. R., WOLF E. S., 1975. Influence of diet on passage rate and apparent digestibility by growing swine. J. Anim. Sci., 40, 486-494.

FAICHNEY G. J., 1975. The effect of formaldehyde treatment of a concentrate diet on the passage of solute and particle markers through the gastrointestinal tract of Sheep. Aust. J. agric. Res., 13, 319-327.

FRANÇOIS E., COMPÈRE R., RONDIA G., 1968. Etude comparative de la vitesse de passage des aliments et des résidus alimentaires non digérés dans le tractus digestif du rat et du mouton. Bull. Rech. agron., Gembloux, 3, 655-688.

FRANÇOIS E., COMPĖRE R., 1971. Mesure du transit gastrointestinal chez le mouton à l'aide des radiolanthanides. Bull. Rech. Agron., Gembloux., 4, 43-61.

FURUYA S., TAKAHASHI S., 1975. Rate of passage of chromic oxide and polyethylene glycol and digestibility in the digestive tract of pigs. Jap. J. zoofech. Sci., 46, 630-641.

ISHIKAWA S., 1966. Reliability of polyethylene glycol as an indicator for digestion studies with swine. Part I. Rate of passage of PEG through the digestive tract. Agric. biol. Chem., 30, 278284.

ISHIKAWA S., FURUICHI Y., 1968. Reliability of PEG as an indicator for digestion studies with swine. Part II. Variation of PEG excretion and estimate of digestibility. Agric. biol. Chem., 32, 1086-1092.

KEYS J. E., DE BARTHE S. V., 1974b. Site and extent of carbohydrate, dry matter, energy and profein digestion and the rate of passage of grain diets in swine. J. Anim. Sci., 39, 57-62. 
LAPLACE J. P., TOMASSONE R., 1970. Evacuation gastro-duodénale chez le porc. Fistulation chronique par voie thoracique extra-pleurale ; recherche d'une technique d'analyse mathématique de l'évacuation. Ann. Zoofech., 19, 303-332.

LAWRENCE T. L. J., 1970a. Some effects of including differently processed barley in the dief of the growing pig. 1. Growth rate, food conversion efficiency digestibility and rate of passage through the gut. Anim. Prod., 12, 139-150.

LAWRENCE T. L. J., 1970b. Some effects of including differently processed barley in the diet of the growing pig. 2. In vivo gastric $\mathrm{pH}$ changes. Anim. Prod., 12, 151-163.

LEVENSPIEL O., 1974. Chemical reaction engineering, 213-315, $2^{\circ}$ Ed., John Wiley and Sons, Inc. New York, London, Sydney, Toronto.

MANER J. H., POND W. G., LOOSLI S. K., LOWREY K. S., 1966. Effect of isolated soybean protein and casein on the gastric $\mathrm{pH}$ and rate of passage of food residues in baby pigs. J. Anim. Sci., 21, 48-52.

MANNERS M. J., KIDDER D. E., 1967. The destruction of polyethylene glycol in gut contents. Proc. Nutr. Soc., 26, 25 (Abstr.).

PONCET C., 1976. Utilisation du cérium 141 comme marqueur de la phase solide des contenus digestifs chez le ruminant. I. Conditions de fixation sur les aliments et comportement dans le contenu de rumen in vivo. Ann. Biol. anim. Bioch. Biophys., 16, 731-739.

RÉRAT A., 1971. Mise au point d'une méthode quantitative d'étude de l'absorption chez le porc. Ann. Biol. anim. Bioch. Biophys., 11, 277-279.

THEWIS A., FRANÇOIS E., THILL N., 1975. Le transit gastrointestinal chez le ruminant mesuré à l'aide des radiolanthanides. Signification des paramètres de la phase exponentielle des courbes de concentration fécale en traceur. Bull. Rech. agron., Gembloux, 10, 307-320.

THEWIS A., FRANÇOIS E., DEBOUCHE C., THIELEMANS M.-F., 1976. Utilisation des radiolanthanides dans la détermination du transit gastrointestinal chez les petits ruminants. Comparaison des techniques directe (abattage) et indirecte. Ann. Zootech., 25, 373-385. 